\title{
LOS HETERÓCLITOS INDOEUROPEOS EN -i/n-: PROPUESTA DE RECLASIFICACIÓN
}

We call -i/n- Heteroclits five nouns which present an alternance in this way: an $i$-stem of N.A.sg. (or masculine gender) is opposed to an $n$-stem of oblique cases (or feminine gender). In four of them this system is used for the opposition of case; this formation appears only in O.I.: ákși, etc. In the remaining nouns, the -i/n-alternance serves for the opposition of gender: O.I. m. páti-/ f. pátmi-, and related forms. The author reviews the etymology of all these nouns: there are phonetic reasons to suppose that the alternance $-i / n$ - is apparent; in all cases they are laringeal stems, as it is justified by comparative material. In short, this inflexional type must be reduced to the heteroclitic $-\phi / n-$ stem.

0.1. Defínimos los heteróclitos en -i/n- como aquellos nombres que, en varias lenguas indoeuropeas, oponen un tema en - $i$ que caracteriza su nominativo-acusativo singular o el género masculino, a un tema en $-n$, sobre el que se forman los casos oblicuos o el género femenino.

0.2 . No vamos a tratar el complicado tema del porqué de dicha alternancia, llamada heteróclisis, bien testimoniada en las lenguas indoeuropeas '. Según nuestra definición, heteróclisis es la oposición de dos temas distintos formados sobre la misma raiz que son utilizados formalmente para oponer, dentro del paradigma de un nombre, dos categorías, ya sea la de caso, la de número o la de género. Recordaremos que los tipos de heteróclisis que realmente están atestiguados en las diferentes lenguas de la familia indoeuropea son cinco:

Un tipo en $-r / n$-, bien conocido, que sustituye el tema en $-r$ que caracteriza su nominativo-acusativo por un tema en $-n$ - en los casos oblicuos. Es el tipo ai. $\overline{\bar{u}}$ dhar, gen. $\dot{\bar{u}}$ dhnas.

Un apartado especial lo ocupa un único nombre de alternancia $-l / n-$ : av. nom. sing. hvar, gen. sing. $x^{2}$ ว̄ng 'sol'.

1 Este trabajo forma parte de un estudio más completo sobre este problema: J. A. Álvarez-Pedrosa, La heteróclisis en la declinación nominal de las lenguas indoeuropeas: Función y origen. Tesis doctoral, Oviedo 1988 (inédita), dirigida por la Dra. J. Mendoza. 
El tercer tipo de alternancia, en $-\phi / n-$, presenta un nom.-ac. en tema puro, opuesto a los casos oblicuos que añaden sus desinencias regulares a un tema en - $n$, como ai. ás, gen. āsnás.

El cuarto es el que nos ocupa, el tipo en $-i / n-$.

Finalmente un tipo de alternancia en $-r / \phi$-, exclusivo del hetita, sirve para formar abstractos por medio de un sufijo alternante: nom.-ac. -war, gen. -waš.

0.3. Hay manuales y monografias que hablan de otros tipos de alternancias ${ }^{2}$, pero ninguno de ellos está atestiguado en las lenguas históricas, por lo que su inclusión en nuestra clasificación la enturbiaría en vez de clarificar el ya oscuro fenómeno de la heteróclisis.

Del mismo modo, hay autores que recurren a la heteróclisis para explicar las diferencias que se observan en la formación de palabras entre las distintas lenguas ides. ${ }^{3}$ Consideramos que esto es un problema específico de derivación mientras que la heteróclisis es un fenómeno de flexión: es una alternancia que se produce en el paradigma de una misma palabra.

Por ello renunciamos a tratar en este mismo estudio el problema de

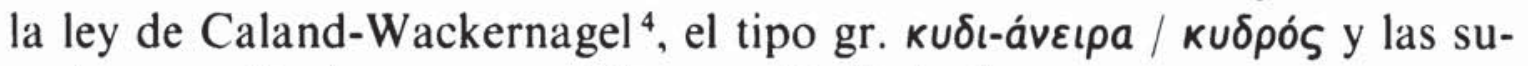
cesivas ampliaciones acometidas por $\mathrm{F}$. Bader ${ }^{5}$.

1. Presentan alternancia $-i / n$ - las siguientes palabras:

1.1. Cuatro neutros del antiguo indio: ákși, gen. akșnás 'ojo'; ásthi, gen. asthnás 'hueso'; dádhi, gen. dadhnás 'leche ácida' y sákthi, gen. sakthnás 'muslo'. En ellos la alternancia descrita sirve para oponer la categoría de caso.

1.2. En otro nombre atestiguado en varias lenguas, la alternancia

${ }^{2}$ A partir de H. Pedersen, «r-n-Stämme», $K Z$ 32, 1893, pp. 240-72, se habla de heteróclitos en $-s / n-,-u / n-,-\bar{a} / n-;$ en el mismo sentido $\mathrm{G}$. Bonfante, "Sobre la función de la heteróclisis en la formación de los temas nominales indoeuropeos", EMERITA 3 , 1935, pp. 257-76; v. también H. Reichelt, "Der sekundäre ablaut. Basenstörung», $K Z$ 39, 1906, p. 66, que incorpora el elemento - $m-;$ H. Petersson, Studien über die indogermanischen Heteroklisie, Lund 1921; Zur Kenntnis der idg. Heteroklisie, Lund 1922, añade $-k,-g,-d,-t,-b$ y los diptongos $-e i,-o i,-e u,-o u,-e r$ y $-o r$.

${ }^{3}$ Sobre todo E. Benveniste, Origines de la formation des noms en indo-curopéen, París 1935.

${ }^{4}$ W. Caland, "Beiträge zur Kenntnis des Avesta", $K Z$ 31, 1892, pp. 256-73; "Beiträge zur Kenntnis des Avesta», $K Z$ 32, 1893, pp. 589-95; J. Wackernagel, Vermischte Beiträge zur griechische Sprachkunde, Basilea 1897.

5 F. Bader, Suffixes grecs en $-\mathrm{m}-:$ Recherches comparatives sur l'heteroclisie nominale, Paris 1974; "Adjectives verbaux hétéroclitiques $\left({ }^{*}-i /{ }^{*}-n t-,{ }^{*}-u-\right)$ en composition nominale», $R P h 49,1975$, pp. 19-48. En la misma línea está el trabajo de J. Haudry, Préhistoire de la flexion nominale indo-européenne, Lyon 1982. 
sirve para oponer género: ai. masc. páti- / fem. pátnī, av. masc. paiti- /

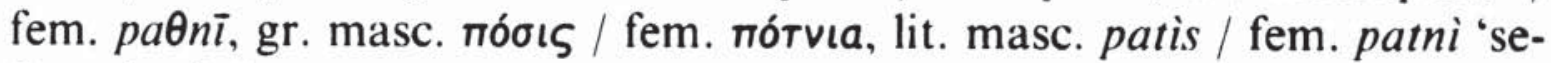
ñor, dueño'.

En el grupo báltico y verosímilmente a imitación de esta palabra se forman las refecciones masc. lit. viešis let. viesis 'huésped' / fem. lit. viešni let. viešna 'huéspeda', sobre todo si tenemos en cuenta que esta palabra aparece en composición en lit. masc. vieš-pãtıiss fem. viẽš-patni 'señor y señora de la casa' 6 .

Es posible que sobre este modelo se forme también el let. masc. atraĩtis 'viudo' fem. atraĩkne 'viuda', en donde el grupo $-k n$ - del femenino procederá de ${ }^{*}$-tn-?

2. Conviene, no obstante, que hagamos una revisión etimológica de todas estas palabras, ya que todas ellas parecen tener un alargamiento laringal.

2.1. Ai. ásthi 'hueso' tiene como correlatos nombres de tema en

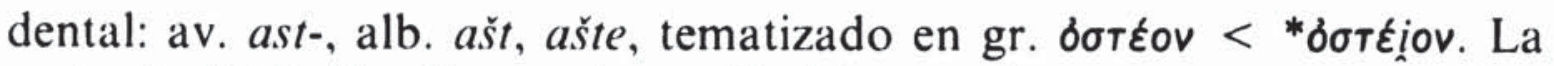
aspirada del indio sólo resulta explicable cuando se compara con el het. haštai- 'hueso'. Hay una cierta unanimidad en explicar los temas en -ai- del het. como temas en ${ }^{*} \mathrm{H}_{2}{ }^{8}$, asi como en reconocer que las sordas aspiradas del indio son resultado de sorda más laringal ${ }^{9}$. Por tanto hay motivos razonables para reconstruir un alargamiento laringal para esta palabra. Esto arrojaría luz sobre el problemático lat. $\check{s}$, $\check{s} s-i s$, que es directamente comparable con luv. hašša-: el resultado -ss- que tantos quebraderos de cabeza ha dado a los etimologistas y fonetistas latinos no sería una excepción del resultado del grupo ${ }^{*}$-st- sino el resultado específico del grupo ${ }^{*}$-st $H-$, al igual que en luv. hašša-.

2.2. Ai. sákthi 'muslo' no tiene correlatos claros fuera del indo-iranio: av. hahti-, mpers. haht, kot. hutä ${ }^{10}$. Sin embargo, el hecho de que

' E. Fraenkel, Litauisches etymologisches Wörterhuch, Heidelberg/Gotinga 1962, p. 1245.

7 V. Pisani, "Appunti di etimologia e morfologia dell'antico indiano", $R A L$ serie 6. ${ }^{a}$, vol. $3 .{ }^{\circ}, 1927$, pp. 429-31.

" E. P. Hamp, "Indo-European nouns with Laryngeal Suffix», Word 9, 1953, pp. 135-41; J. Gil, "Los temas nominales en laringal», EMERITA 37, 1969, p. 401.

"La propuesta arranca del formulador de la teoría laringal: F. de Saussure, "Contribution à l'histoire des aspirées sourdes", BSL 7, 1892, p. 118; v. la historia de la cuestión y el proceso de creación de alófonos aspirados de las oclusivas sordas ides. en F. Villar, "El problema de las sordas aspiradas indoeuropeas", RSEL 1, 1971, pp. 129-60. También reconoce una etimología con laringal R. S. P. Beekes, "Indo-European neuters in -i», Festschrift Hoenigswald, Tubinga 1987, pp. 45-56.

${ }^{10}$ M. Mayrhofer, Kurzgefaßtes etymologisches Wörterbuch des Altindischen. Heidelberg 1956, Bd. III, p. 412. 
la raiz testimonie una dental sorda aspirada y que dé lugar en indio tanto al neutro sákthi como al femenino sákthī" hace plausible la explicación de que se trata de un tema alargado mediante ${ }^{*}-H_{2}$.

Es posible que en el alargamiento laringal en la palabra para 'muslo' haya tenido alguna influencia la palabra ásthi 'hueso' ${ }^{12}$.

2.3. Ai. dádhi 'leche ácida' se ha interpretado tradicionalmente como un tema reduplicado *de-dh- de la raíz del verbo ai. dháyati 'mamar' ${ }^{13}$. Esta misma raiz forma el verbo het. tittiya-, luv. tittai- del mismo significado, siendo ambos verbos susceptibles de explicación laringal $^{14}$. Por tanto podemos reconstruir razonablemente un alargamiento laringal para ai. dádhi.

2.4. Ai. ákși 'ojo' no presenta tantos puntos de apoyo claros en su etimología para reconstruir un elemento laringal. Con todo, la presencia en ai. ákși y gr. ő $\sigma \sigma \varepsilon<{ }^{*} o k^{u}-i-e$ de un elemento vocálico $-i$ - que no aparece en otras lenguas: lat. oculus, got. augo, arm. akn, toc. B esane, en el propio ai. an-ák 'ciego' ${ }^{15}$, en el gen.-loc. du. akșós, el gen. pl. ák-

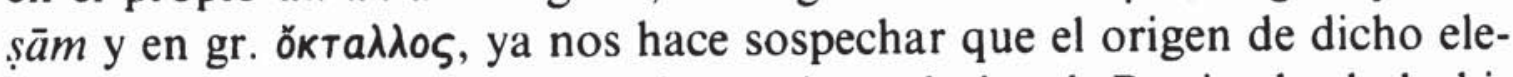
mento vocálico puede ser un alargamiento laringal. Partiendo de la hipótesis tradicional del origen dual de esta palabra ${ }^{16}$, otros autores consideran que la discordancia en la desinencia de nom.-ac. dual ${ }^{*}-\bar{l}$ en ai.

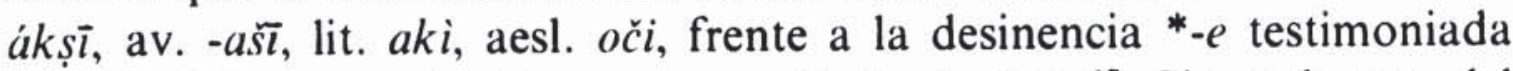
por gr. ö $\sigma \sigma \varepsilon$, es explicable como resultado de ${ }^{*}-H_{1}{ }^{17}$. Sin embargo, del mismo modo que podemos testimoniar vocalizaciones de laringal con

" H. Grassmann, Wörterbuch zum Rig-Veda, Wiesbaden 1872, p. 1440.

12 V. también Beekes, art. cit.

13 Benveniste, op. cit., p. 184; Mayrhofer, op. cit., II, p. 15.

14 F. R. Adrados, Evolución y estructura del verbo indoeuropeo, Madrid 1974, pp. 124-27; Beekes, art. cit., propone también una etimología con laringal, aunque con menor justificación desde nuestro punto de vista.

is V. A. J. Nussbaum, Head and Horn in Indo-European, Berlin/Nueva York 1986, p. 199.

${ }_{16}$ Esta propuesta fue formulada por G. H. M. Mahlow, Die langen Vokale AEO in den europäischen Sprachen, Berlin 1879, p. 102; fue recogida en nuestros días por O. Szemerényi, "The name of "heart"», Donum Balticum. Festschrift Stang, Estocolmo 1971, pp. 515-33. J. Wackernagel, Altindische Grammatik, Gotinga 1929-1930, III, p. 305, alega a esto que el falso corte a partir del dual puede admitirse para áks
y sákthi, pero no para ásthi y dádhi, en donde el dual no ha jugado ningún papel.

17 B. Forssmann, "Nachlese zu $\delta \sigma \sigma \varepsilon », ~ M S S ~ 25,1969$, pp. 39-50; V. I. Georgiev, «Die Herkunft der indoeuropäischen Endungen für Nominativ-Akkusativ-Vokativ Plural Neutrum und Dual», IF 78, 1973, pp. 43-50; "L'origine des désinences du nominatif-accusatif-vocatif duel d'après les données mycéniennes", Colloquium Mycenaeum, Neuchâtel/Ginebra 1979, pp. 341-46; Beekes, art. cit.; N. Oettinger, «Der indogermanische Nominativ Dual aus laringalistischer Sicht», Die Laryngaltheorie, ed. Bammesberger, Heidelberg 1988, pp. 355-59. 
resultado de timbre $-i$ - en gr. (y desde luego en ai.), la vocalización ${ }^{*}-H_{1}$ $>-e$ es dificil de admitir salvo para casos de regularización de paradigmas ${ }^{18}$.

2.5. En el nombre alternante ai. masc. páti- / fem. pátnī etc. ${ }^{19}$ el tema en laringal se puede deducir a partir de alternancias entre vocal larga y vocal breve testimoniadas en la misma raíz, en lat. potittur / potïtur.

3. Es importante señalar que gr. ä\фı no es un heteróclito en $-i / n$ como se viene repitiendo de un modo mecánico en muchos manuales.

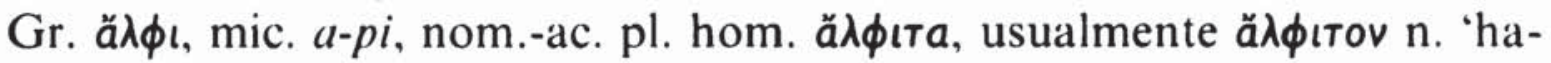

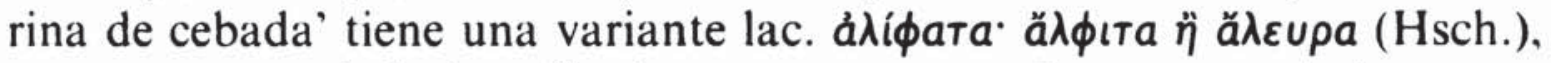
que numerosos helenistas ${ }^{20}$ reinterpretan como el nom.-ac. pl. de un pa-

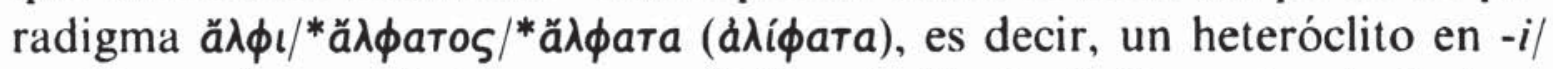

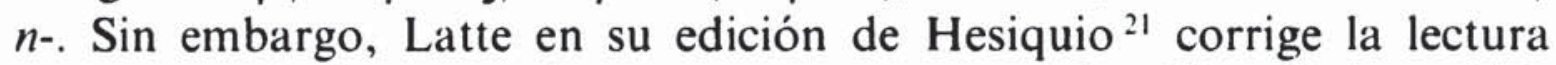

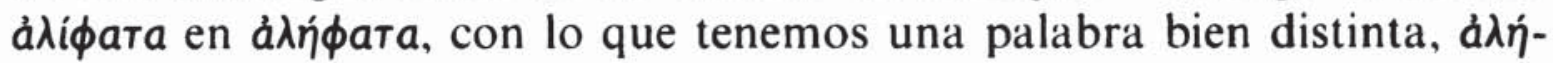

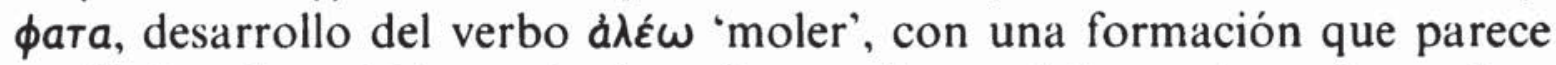
analógica de $\mu$ $\lambda \dot{\phi} \phi a r o s ~ ' a p l a s t a d o$ por la muela', gracias a la cual se explica la variante hom. ádeíфaros ${ }^{22}$.

4. Así pues, parece claro que el elemento $-i$ del nominativo-acusativo de estos cuatro neutros y del masculino de ai. páti- se puede explicar bien como vocalización de una laringal. El hecho de que la laringal vocalice en $-i$ en indio resulta regular. Que vocalice en - $i$ en gr. пótı५ o en lit. patis entra dentro de las posibilidades de vocalización de estos fonemas. De hecho estos nombres han sido incluidos dentro de los temas en laringal por Hamp y J. Gil y parcialmente por Beekes.

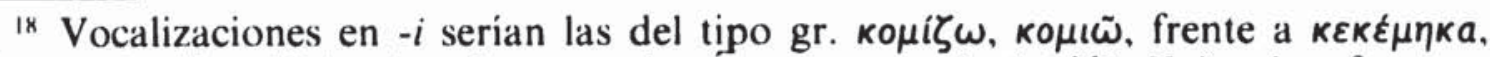

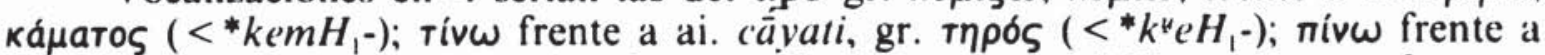

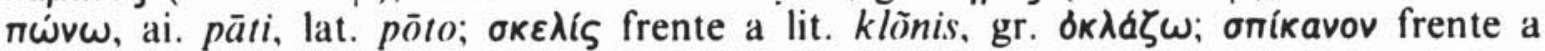
maa. spān, aaa. spanan. La teoría que defiende vocalizaciones $H_{1}>e, H_{3}>0$, que arranca de J. Kurylowicz, Études indo-européennes, Cracovia 1935, p. 44, sólo se

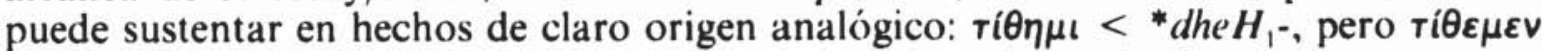
$<{ }^{*} d h H_{1}-$, en donde $H_{1}>e$ por analogía con el paradigma de singular.

${ }_{19} \mathrm{O}$. Szemerényi, Syncope in Greek and Indo-European and the Nature of IndoEuropean Accent, Nápoles 1964, pp. 389-95 explica ai. f. patnī como síncopa de un femenino en -ini *potini ; v. las dudas al respecto en $\mathrm{P}$. Chantraine, Dictionnaire étymologique de la langue grecque. Histoire des mots, París 1980, p. 932.

20 F. Fröhde, "Der lateinische ablaut III", BB 7, 1883, p. 104; H. Ehrlich, "Die

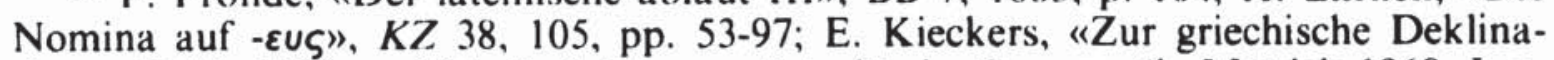
tion", IF 41, 1923, p. 184; E. Schwyzer, Griechische Grammatik, Munich 1968, I, p. 518.

${ }^{21}$ K. Latte (ed.), Hesychii Alexandrini Lexicon, Copenhague 1953-1966, s.u.

22 Chantraine, op. cit. 
Sin embargo el elemento $-i$ ha recibido previamente explicaciones muy variadas desde Bopp en adelante. Podriamos dividir las teorias en tres grupos.

4.1. Hay un grupo de explicaciones que podiamos llamar marginales, como sería la del propio Bopp ${ }^{23}$, quien pensó que la flexión originaria de estos temas era enteramente en $-n$, y que $-i$ era un elemento «adventicio». De Saussure ${ }^{24}$ lo explica como un elemento eufónico motivado por la acumulación de consonantes en los casos del plural, tipo asthi-bhis, etc. J. Schmidt ${ }^{25}$ opina que $-i$ es propio de adjetivos y pronombres, por lo que habría habido una sustantivación de éstos.

4.2. Otros autores ven en - $i$ una marca casual, ya sea de locativo, como piensan Hirt o Haudry ${ }^{26}$, ya sea de dual ${ }^{27}$. Efectivamente, no sería imposible que ai. nom.-ac. sg. ákşi se hubiera originado a partir de

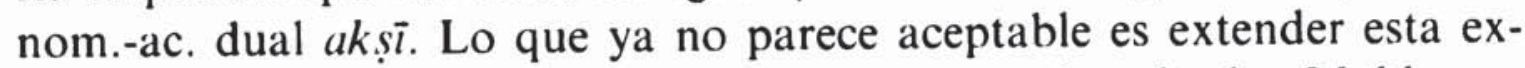
plicación al resto de los neutros en $-i / n$ - como han hecho Mahlow y Szemerényi.

4.3. La mayoría de las explicaciones se orientan en cambio hacia un alargamiento de la raíz. Así por ejemplo Bartholomae, Brugmann, Schulze, o Benveniste ${ }^{2 x}$, quien piensa que $-i$ es un alargamiento en tanto en cuanto que se añade al nominativo-acusativo sin influir sobre el resto de la flexión; también Villar ${ }^{29}$ piensa que se trata de un alargamiento que sirvió para caracterizar el nominativo-acusativo frente al resto de los casos y fue exclusivo del antiguo indio; la morfologización fue incompleta porque no se llegó a sentir esa $-i$ como verdadera desinencia casual portadora del valor nominativo-acusativo y no recibió empleo sistemático.

5. Ahora bien, ¿en qué medida el hecho de que estos nombres tengan un tema en laringal en el nominativo-acusativo clarifica la posición

${ }^{23}$ F. Bopp, Ausführliches Lehrgebäude der Sanskrit-Sprache, Berlin 1827, p. 103 s.; Grammatica critica linguae Sanscritae. Altera emendata editio, Berlín 1832, p. 89 s.

${ }_{24}$ F. de Saussure, Recueil des publications scientifiques, Ginebra 1921, p. 209.

25 J. Schmidt, Die Pluralhildungen der indogermanische Neutra, Weimar 1889, p. 257.

${ }^{26} \mathrm{H}$. Hirt, "Über der Ursprung der Verbalflexion im Indogermanischen», IF 17, 1904-1905, pp. 36-84; Indogermanische Grammatik, Bd. III, Heidelberg 1927, p. 102 s.

27 V. nota 16.

2* Chr. Bartholomae, "Arisches», BB 15, 1889, p. 38; K. Brugmann, Grundriß der vergleichenden Grammatik der indogermanischen Sprachen, Bd. II I, Estrasburgo 1906, pp. 173-75, 577 s.; W. Schulze, Kleine Schriften, Gotinga 1933, p. 79; E. Benveniste, op. cit., p. 86.

${ }_{29}$ F. Villar, Origen de la flexión nominal indoeuropea, Madrid 1974, p. 217. 
de éstos dentro de la clasificación de los heteróclitos que hemos enunciado? El propio Hamp ${ }^{30}$, que ya vio que eran temas en laringal, modifica la clasificación tradicional y sustituye los heteróclitos en $-i / n$ - por temas en $-H / n$-. Beekes ${ }^{31}$, por su parte, niega la existencia de temas en $-i / n-$, que serían neutros en $-i / \phi$ - originados de temas en laringal. Con ello desprecia el testimonio de una lengua, el antiguo indio, en donde estos heteróclitos están testimoniados desde el védico, sin dar una explicación convincente: el hecho de que no tengan correlatos en iranio y las otras lenguas (que no es cierto en el caso de ai. páti-/pátnī) no los hace menos reales. En suma, estos dos autores sustituyen la clasificación tradicional de heteróclitos en $-i / n$ - por otras dos nuevas y distintas.

Nuestra propuesta es, sin embargo, reduccionista: la alternancia - $i$ / $n$ - es aparente: en realidad se trata de temas de alternancia "tema puro" / $-n-$, del tipo que hemos llamado $-\phi / n-$. El tema puro es un tema en laringal que vocaliza en $-i$ mientras que los casos oblicuos o el femenino se forman sobre el tema en -n-, exactamente igual que ai. ás gen. āsnás. La clave que nos da la solución es ver que la laringal del tema en los casos oblicuos y el femenino se encontró en posición interconsonántica y en ese contexto desapareció:

nom. ${ }^{*} d e-d h H>$ ai. dádhi, pero gen. ${ }^{*}$ dedh $(H)-n-o s>$ ai. dadhnás. masc. ${ }^{*} p o t H->$ ai. páti-, pero fem. ${ }^{*} \operatorname{pot}(H)-n-e H_{2}>$ ai. pátnī.

La caida de la laringal provoca que parezca que en estos temas la $-i$ del nom.-ac. o la del masc. es sustituida por la $-n$ de los casos oblicuos o del fem., lo mismo que sucede en los temas en $-r / n$ - como ai. $\dot{\bar{u}} d h a r$ gen. $\bar{u} d h n a s$. En realidad, el tema en $-n$ - de los casos oblicuos y del fem. se añade al tema de los casos rectos, no lo sustituye, por lo que la caída de la laringal intervocálica ha enmascarado la flexión alternante originaria de estos temas, que, en realidad, fue del tipo $-\phi / n-$.

\section{Juan Antonio Álvarez-Pedrosa}

\footnotetext{
30) Hamp, art. cit.

3 Beekes, art. cit.
} 